\title{
Soil fertility status and nutrients provided to spring barley (Hordeum distichon L.) by pig slurry
}

\author{
Melisa Gómez-Garrido ${ }^{1 *}$,Silvia Martínez-Martínez ${ }^{1}$, Ángel Faz Cano ${ }^{1}$,Asuman Büyükkılıç-Yanardag ${ }^{1}$, \\ and Joselito M. Arocena ${ }^{2}$
}

Nutrient recycling using pig slurry is a common agricultural practice to manage the ever-increasing amounts of wastes from the pig industry. This study was conducted in the southeast of Spain to quantify the enrichments in major (N, P, K, $\mathrm{Mg}$ ) and minor ( $\mathrm{Zn}, \mathrm{Fe}, \mathrm{Cu}$, and $\mathrm{Mn}$ ) nutrients in soils amended with $\mathrm{D} 1-170 \mathrm{~kg} \mathrm{~N}^{-1}$ (European Union legislated dose) or D2-340 kg N ha-1, and understand the influence of pig slurry on yield and nutrient uptake in two crop seasons of spring barley (Hordeum distichon L.) Compared to control, D2 increased $\mathrm{NO}_{3}{ }^{-}-\mathrm{N}$ by $11.4 \mathrm{X}$ to $109 \mathrm{mg} \mathrm{kg}^{-1}$, Olsen-P by $6.9 \mathrm{X}$ to 423 mg kg-1 , exchange $\mathrm{K}\left(2.5 \mathrm{X}\right.$ to $\left.1.6 \mathrm{cmol}_{+} \mathrm{kg}^{-1}\right), \mathrm{Mg}\left(1.7 \mathrm{X}\right.$ to $\left.1.8 \mathrm{cmol}_{+} \mathrm{kg}^{-1}\right)$, diethylene-triamine pentaacetic acid (DTPA)-Zn ( $94 \mathrm{X}$ to $18.2 \mathrm{mg} \mathrm{kg}^{-1}$ ), and $\mathrm{Fe}\left(2 \mathrm{X}\right.$ to $11.3 \mathrm{mg} \mathrm{kg}^{-1}$ ). Available $\mathrm{NO}_{3}^{-}-\mathrm{N}$, Olsen-P, and DTPA-Zn have the best correlations with crop yield and nutrient uptake. These results indicate that the assessment of soil fertility status at 1-mo after pig slurry addition provides a good indicator for potential yield and uptake of barley. However, it is suggested that leachates should be monitored to effectively manage potential releases of nitrate and phosphate into the environment.

Key words: Biomass yield, field experiment, microplots, optimal timing.

\section{INTRODUCTION}

Intensive pig production generates large quantities of organic wastes such as pig slurry (PS). Global pig production produced more than 1.5 billion tons of pig manure in 2005 (Kuligowski et al., 2010). In Spain, the pig industry released an estimated 71 million $\mathrm{m}^{3}$ of waste residues in 2008 (MARM, 2008) and in the last $10 \mathrm{yr}$, hog farms produce 4 million tons of PS every year (Real Decreto 324/2000, 2000; Eurostat, 2010; MARM, 2010). The disposal of large quantities of pig wastes presents several environmental challenges and economic opportunities for producers, environmental scientists, engineers as well as government regulators (Martínez and Burton, 1998).

Agricultural utilization of pig wastes as organic fertilizer is a common practice in many pig-producing countries and as soil reclamation amendment in anthropogenically-altered environments (e.g., industrial and mine tailing deposits). Pig slurry is a valuable source of $\mathrm{N}$ for maize and other crops (Daudén and Quílez, 2004; Biau et al., 2012) and soil organic matter (SOM) for the

\footnotetext{
${ }^{1}$ Universidad Politécnica de Cartagena, Departamento de Ciencia y Tecnología Agraria, Paseo Alfonso XIII, 52, 30203 Cartagena, Murcia, España.

*Corresponding author (melisa.gomezgarrido@hotmail.com).

${ }^{2}$ University of Northern British Columbia, College of Science and Management, 3333 University Way, Prince George, British Columbia, Canada V2N4Z9.

Received: 7 June 2013.

Accepted: 28 November 2013.

doi:10.4067/S0718-58392014000100012
}

reclamation of mine wastes deposits (e.g., Zanuzzi et al., 2009; Zornoza et al., 2012). Use of PS in agriculture increases the fertility status by supplying essential nutrients such as $\mathrm{N}$ and $\mathrm{P}$ as well as improves physical properties of soils (Ayuso et al., 1996; Gilly and Eghball, 2002; Soumare et al., 2003; Biau et al., 2012). Utilization of PS as $\mathrm{N}$ source (148-590 kg N ha-1) increased the yield of willow plantation in eastern Canada by $14.4-24.4 \%$ (30.3-32.9 t ha $^{-1}$ ) compared to Control plots (Cavanagh et al., 2011). Furthermore, for corn fertilized with PS in northeast Spain, biomass, yield and $\mathrm{N}$ uptake were similar to plots treated with mineral fertilizer (Daudén and Quílez, 2004). Other studies suggested that crop responses to organic manures and fertilizer treatments were dependent on site location, soil type or year of application (Gagnon et al., 1997; Stevenson et al., 1998). Yagüe et al. (2012) reported a $74 \%$ increased in water-stable aggregates due to application of PS in soils of northeastern Spain.

The main objective of this study was to understand agricultural benefits associated with PS application to agricultural crops in the vicinity of intensive pig industry in a typical Mediterranean environment. Specifically, our purposes were (1) to monitor changes in amounts of major (N, P, K, and $\mathrm{Mg}$ ) and minor ( $\mathrm{Zn}, \mathrm{Fe}, \mathrm{Cu}$, and $\mathrm{Mn}$ ) nutrients in soils, and (2) quantify the yield and uptake of spring barley (Hordeum distichon L.) in response to two applications of PS in two doses into an agricultural soil in southeast Spain. The information reported in this research will be useful to farmers in relation to the amount and optimal timing of soil PS applications to barley cultivation in semiarid Mediterranean environments. 


\section{MATERIALS AND METHODS}

\section{Study area and experimental design}

The experiments were conducted in Doña Inés, Murcia

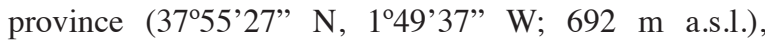
southeast Spain. The study site, with $3.4 \%$ slope, has semiarid Mediterranean climate with mean annual temperature of $15{ }^{\circ} \mathrm{C}$, a mean annual precipitation of $300 \mathrm{~mm}$ and Typic Haplocalcid (USDA, 2010) is the dominant soil. Selected properties from 42 samples collected prior to application of treatments are as follows: $\mathrm{pH} 8.2$, electrical conductivity (EC) $0.27 \mathrm{dS} \mathrm{m}^{-1}$, total $\mathrm{N} 609 \mathrm{mg} \mathrm{kg}{ }^{-1}, 62 \mathrm{mg}$ Olsen-P kg-1, 9 cmol $_{+} \mathrm{kg}^{-1}$ cation exchange capacity (CEC), $0.5 \mathrm{cmol}_{+} \mathrm{kg}^{-1}$ exchangeable $\mathrm{K}$, and $75.1 \% \mathrm{CaCO}_{3}$.

A completely randomized design experiment consisting of two doses of PS and control applied to two crops of spring barley was conducted for 2 yr (March 2009-June 2011) in a piece of vacant land with history of cereal cultivations without any past addition of inorganic fertilizers. The first barley crop was planted in December 2009 and harvested in June 2010 while the second season started in January 2010 and ended in July 2011. The three treatments: Control without PS, D1 $170 \mathrm{~kg} \mathrm{~N} \mathrm{ha}^{-1} \mathrm{yr}^{-1}$, D2 $340 \mathrm{~kg} \mathrm{~N} \mathrm{ha}^{-1} \mathrm{yr}^{-1}$, were applied on a $25 \mathrm{~m}^{2}$ plot and separated from each other by a 3-m wide corridor. First PS applications were added on October 2009 using $1.2 \mathrm{~g}$ $\mathrm{N} \mathrm{L}^{-1}$ or $430 \mathrm{~L}$ PS for D1 and $860 \mathrm{~L}$ PS for D2 plots. After 1 yr (October 2010), we repeated PS applications using 300 and $600 \mathrm{~L}$ of PS in D1 and D2 plots, respectively. The applied PS materials were collected from an intensive pig farm maternity located in the surroundings ("Doña Inés" farm, Lorca, Murcia) and plowed into 0-30 $\mathrm{cm}$ surface layer of soils in the study area. There were three replicated plots for each D1 and D2 treatments but due to limited logistics and uniformity of the land area, there was only one plot in the control treatment. However, three random sampling points were used in each plot throughout the experiments.

Selected properties of PS amendments used in the experiment include: $\mathrm{pH} 7.2$ (2009) and 7.6 (2010), total N 1.0 (2009) and $1.4 \mathrm{~g} \mathrm{~N} \mathrm{~L}^{-1}$ (2010), total P 118 (2009) and $184 \mathrm{~g} \mathrm{~N} \mathrm{~L}^{-1}$ (2010) (Table 1).

\section{Soil sample collection and analyses}

Soil samples were collected every 3-5-mo throughout the duration of the experiment. We designated the five soil sampling events with respect to the application of PS and cultivation of the two barley crop seasons:

(A) 2009-2010 Barley Crop Season: Pre-Crop 1 (PC1) November 2009, 1-mo after the first PS application; Crop Season 1 (CS1) - March 2010, maturity stage of barley.

(B) 2010-2011 Barley Crop Season: Residual (RES) July 2010, prior to second application of PS; Pre-Crop 2 (PC2) December 2010, 1-mo after the second application of PS; Crop Season 2 (CS2) June 2011, maturity stage of barley.
Table 1. Mean and standard error (parentheses) of selected properties of pig slurry collected from a pig farm maternity located in Lorca, Murcia, Spain, used in the experiment, $n=3$.

\begin{tabular}{lcc}
\hline Properties & 2009 Pig slurry & 2010 Pig slurry \\
\hline Density, g mL & $1.00(0.00)$ & $0.90(0.03)$ \\
pH & $7.2(0.02)$ & $7.6(0.03)$ \\
$\mathrm{EC}, \mathrm{mS} \mathrm{cm}^{-1}$ & $16.1(0.03)$ & $16.7(1.1)$ \\
Moisture, \% & $95(0.73)$ & $96(0.87)$ \\
Total N, g L & $1.41(0.02)$ \\
$\mathrm{NH}_{4}^{+}-\mathrm{N}, \mathrm{g} \mathrm{L}^{-1}$ & $1.00(0.04)$ & $1.18(0.01)$ \\
$\mathrm{P}, \mathrm{mg} \mathrm{L}^{-1}$ & $0.81(0.04)$ & $184(14)$ \\
$\mathrm{Mg}, \mathrm{mg} \mathrm{L}^{-1}$ & $118(5)$ & $145(8.9)$ \\
$\mathrm{Ca}, \mathrm{mg} \mathrm{L}^{-1}$ & $71(15.3)$ & $141(7.8)$ \\
$\mathrm{K}, \mathrm{mg} \mathrm{L}^{-1}$ & $113(4.9)$ & $1020(55)$ \\
$\mathrm{Na}, \mathrm{mg} \mathrm{L}^{-1}$ & $836(7)$ & $1194(17)$ \\
$\mathrm{Zn}, \mathrm{mg} \mathrm{L}^{-1}$ & $868(11)$ & $3.9(1.16)$ \\
$\mathrm{Fe}, \mathrm{mg} \mathrm{L}^{-1}$ & $3.4(0.16)$ & $6.5(1.48)$ \\
$\mathrm{Cu}, \mathrm{mg} \mathrm{L}^{-1}$ & $3.8(0.41)$ & $0.50(0.17)$ \\
$\mathrm{Mn}, \mathrm{mg} \mathrm{L}^{-1}$ & $0.67(0.02)$ & $0.69(0.10)$ \\
\hline
\end{tabular}

Samples prior to PS application (March and July 2009) were collected to determine the initial characteristics of the soil. Each sample was a composite sample from three random points in every plot at surface $(0-30 \mathrm{~cm})$ and subsurface $(30-60 \mathrm{~cm})$ soils. Each soil sample was air-dried and sieved $<2 \mathrm{~mm}$ before analyses. Soil data reported in this research are limited to $0-30 \mathrm{~cm}$ results because our focus was to report the benefits of PS to the growth of spring barley. The $30-60 \mathrm{~cm}$ soil samples are primarily for the evaluation of potential $\mathrm{NO}_{3}{ }^{-}$leaching and results will be reported elsewhere.

Soil $\mathrm{pH}$ was measured in de-ionized water $(1: 2.5 \mathrm{w} / \mathrm{v})$ with a pH meter (GLP 21, Crison, Barcelona, Spain) while EC was measured in a 1:5 soil:water solution using a ECmeter (GLP 31, Crison, Barcelona, Spain). Organic carbon (OC) was determined by $\mathrm{K}_{2} \mathrm{Cr}_{2} \mathrm{O}_{7}$ oxidation (Walkley and Black, 1934) while total $\mathrm{N}$ was estimated using the Kjeldahl method (Duchaufour, 1970). Concentrations of $\mathrm{NH}_{4}{ }^{+}-\mathrm{N}$ and $\mathrm{NO}_{3}{ }^{-}-\mathrm{N}$ were determined by UV-visible spectrophotometry (spectrophotometer UVmini-1240, Shimadzu, Kyoto, Japan) in a $2 \mathrm{M} \mathrm{KCl}$ extract (1:10 w/v) (Keeney and Nelson, 1982; Sempere et al., 1993; Kandeler et al., 1999). Available $P$ was measured using the sodium-bicarbonate extraction-based Olsen-P method (Burt, 2004) while the bio-available minor elements ( $\mathrm{Zn}$, $\mathrm{Fe}, \mathrm{Cu}$, and $\mathrm{Mn}$ ) were estimated using the diethylenetriamine pentaacetic acid (DTPA) method (Lindsay and Norvell, 1978).

\section{Plant sample collection and analyses}

At the end of each crop season, all barley plants within a randomly-selected $1 \mathrm{~m}^{2}$ area in each plot were collected to estimate biomass and uptake of major and minor elements. Total biomass (ear + grain) was determined in the field and five representative barley plants were chosen and taken to the laboratory for length, weight, moisture content, major and minor elements uptake measurements. Plant samples were dried and ground prior to laboratory analyses. 
The total uptake of major and minor elements was determined in plant samples using the procedure recommended by Madrid et al. (1996). Approximately $0.7 \mathrm{~g}$ ground sample (ear and grain) were dried at $480{ }^{\circ} \mathrm{C}$ for $24 \mathrm{~h}$ and digested using $0.6 \mathrm{~N} \mathrm{HNO}_{3}$. Total $\mathrm{Na}$ and $\mathrm{K}$ were measured by flame photometry while $\mathrm{Ca}, \mathrm{Mg}, \mathrm{Zn}$, $\mathrm{Fe}, \mathrm{Cu}$, and $\mathrm{Mn}$ were determined by atomic absorption technique (AAnalyst 800, PerkinElmer Precisely, Shelton, Connecticut, USA). Total P in plant sample was determined from the $\mathrm{HNO}_{3}$-digest using the ammonium molybdate method (Jeffery et al., 1989).

Total $\mathrm{N}$ in plant samples was determined by the Kjeldahl method (Duchaufour, 1970). Nitrate content in plant sample was estimated after reaction of $0.45-0.50 \mathrm{~g}$ ground sample with $25 \mathrm{~mL}$ distilled water. The $\mathrm{NO}_{3}{ }^{-}-\mathrm{N}$ in the extract was photometrically (photometer PF-11, Macherey-Nagel, Düren, Germany) estimated using the 2,6-dimethyl-phenol in a mixture of $\mathrm{H}_{2} \mathrm{SO}_{4}-\mathrm{H}_{3} \mathrm{PO}_{4}$ acid method test Nanocolor (APHA, AWWA, WPCF, 1992).

\section{Statistical analyses}

A two-factor design (i.e., amendments - Control, D1, D2, and sampling times - PC1, CS1, RES, PC2, and CS2) was used to evaluate temporal changes to soil properties due to PS applications. The Fisher LSD test was conducted to compare means of significantly different treatments. Correlations between various forms of soil $\mathrm{N}$, available $\mathrm{P}$, exchangeable $\mathrm{K}, \mathrm{Mg}$, $\mathrm{Na}$, bio-available $\mathrm{Zn}, \mathrm{Fe}, \mathrm{Cu}$, and $\mathrm{Mn}$ and plant uptake of major and minor nutrients and biomass yields were also determined. All statistical analyses were conducted using Statistica version 10 (StatSoft Inc., 2011).

\section{RESULTS}

\section{Soil properties}

All soil properties but total $\mathrm{N}$ exhibited significant interactions between treatments and times of sample collection (Tables 2-5). Soil pH ranged from 7.4 (PC2-D2) to 8.6 (CS1-Control) while EC in D2 plots in the PC2 and CS2 sampling periods were significantly the highest values at 0.40 and $0.34 \mathrm{dS} \mathrm{m}^{-1}$, respectively (Table 2). Calcium carbonate contents varied from 52\% in PC1-D2 plot to $68 \%$ in PC1-control. The amounts of Olsen-P were generally higher in D1 and D2 than control plots at all sampling events. Olsen-P in control plots ranged from 181 to $191 \mathrm{mg} \mathrm{P} \mathrm{kg}^{-1}$ while the low and high limits in D1 and D2 were $80-260$ and $243-423 \mathrm{mg} \mathrm{P} \mathrm{kg}^{-1}$, respectively (Table 2).

The sum of $\mathrm{NO}_{3}{ }^{-}-\mathrm{N}$ and $\mathrm{NH}_{4}{ }^{+}-\mathrm{N}$ constituted $<10 \%$ compared to total $\mathrm{N}$ (Table 3). In PC1 samples, $\mathrm{NH}_{4}{ }^{+}-\mathrm{N}$ was greater than $\mathrm{NO}_{3}{ }^{-} \mathrm{N}$ but the reverse was true for samples during the PC2 collection especially for D1 and $\mathrm{D} 2$ treatments. Total $\mathrm{N}$ did not show interactions between time of sampling and amounts of PS additions. Organic C contents $\left(\mathrm{g} \mathrm{C} \mathrm{kg}^{-1}\right)$ ranged from 8.3 in PC1-D1 samples to 16.2 in $\mathrm{CS} 2-\mathrm{D} 2$ plots while $\mathrm{C} / \mathrm{N}$ ratio remained $<25$
Table 2. Changes to mean and standard error (parentheses) of $\mathbf{p H}$ in $\mathrm{H}_{2} \mathrm{O}$, electrical conductivity (EC), $\mathrm{CaCO}_{3}$, and Olsen-P at five sampling periods during two consecutive cropping seasons of spring barley grown in agricultural soil amended with two doses (D1 $=170 \mathrm{~kg} \mathrm{~N} \mathrm{ha}^{-1} \mathrm{yr}^{-1} ; \mathrm{D} 2=340 \mathrm{~kg} \mathrm{~N} \mathrm{ha}^{-1} \mathrm{yr}^{-1}$ ) and two applications (October 2010 and 2011) of pig slurry in Lorca, Spain, $n=$ number of observations.

\begin{tabular}{|c|c|c|c|c|c|}
\hline \multirow[b]{2}{*}{ Treatment } & \multirow[b]{2}{*}{$\mathrm{n}$} & \multirow[b]{2}{*}{$\mathrm{pH}$ in $\mathrm{H}_{2} \mathrm{O}$} & \multirow{2}{*}{$\begin{array}{c}\text { EC } \\
\mathrm{dS} \mathrm{m}^{-1}\end{array}$} & \multirow{2}{*}{$\frac{\mathrm{CaCO}_{3}}{\%}$} & \multirow{2}{*}{$\begin{array}{l}\text { Olsen-P } \\
\mathrm{mg} \mathrm{kg}^{-1}\end{array}$} \\
\hline & & & & & \\
\hline \multicolumn{6}{|c|}{ Pre-crop 1 (November 2009) } \\
\hline Control & 3 & $8.4(0.12) \mathrm{de}^{\dagger}$ & $0.17(0.01) \mathrm{ab}$ & $68(5.80) \mathrm{g}$ & $191(36.1) b c$ \\
\hline D1 & 9 & $8.2(0.06) \mathrm{d}$ & $0.19(0.02) \mathrm{ab}$ & $54(1.70) \mathrm{ab}$ & 80 (21.9)a \\
\hline D2 & 9 & $8.3(0.04) \mathrm{de}$ & $0.26(0.01) \mathrm{cd}$ & $52(0.90) \mathrm{a}$ & 355 (2.0)fg \\
\hline \multicolumn{6}{|c|}{ Crop season 1 (March 2010) } \\
\hline Control & 3 & $8.6(0.08) \mathrm{e}$ & $0.14(0.02) \mathrm{a}$ & 57 (0.50)bcd & $181(3.4) b c$ \\
\hline D1 & 9 & $8.5(0.04) \mathrm{e}$ & $0.20(0.02) \mathrm{ab}$ & $59(1.40)$ cde & $230(23.1) \mathrm{cd}$ \\
\hline D2 & 9 & $8.4(0.09) \mathrm{de}$ & $0.20(0.01) \mathrm{ab}$ & $54(0.40) \mathrm{ab}$ & 297 (28.6)def \\
\hline \multicolumn{6}{|c|}{ Residual (July 2010) } \\
\hline Control & 3 & $8.4(0.15) \mathrm{de}$ & $0.20(0.04) \mathrm{ab}$ & 57 (0.55)bcd & 187 (1.3)bc \\
\hline D1 & 9 & $8.5(0.05) \mathrm{e}$ & $0.21(0.03) \mathrm{abc}$ & $53(1.40) \mathrm{a}$ & $225(16.5) \mathrm{cd}$ \\
\hline D2 & 9 & $8.5(0.04) \mathrm{e}$ & $0.24(0.01) b c$ & $57(1.20) b c$ & $243(16.6) \mathrm{cd}$ \\
\hline \multicolumn{6}{|c|}{ Pre-crop 2 (December 2010) } \\
\hline Control & 3 & $8.1(0.17) \mathrm{cd}$ & $0.16(0.01) \mathrm{a}$ & $60(0.54) c-f$ & $186(2.7) b c$ \\
\hline D1 & 9 & $7.9(0.06) \mathrm{bc}$ & $0.29(0.02) \mathrm{de}$ & $61(0.90)$ def & 260 (22.6)cde \\
\hline D2 & 9 & $7.4(0.21) \mathrm{a}$ & $0.40(0.02) \mathrm{f}$ & $61(0.68)$ def & $423(58.8) \mathrm{g}$ \\
\hline \multicolumn{6}{|c|}{ Crop season 2 (June 2011) } \\
\hline Control & 3 & $8.1(0.09) \mathrm{cd}$ & $0.15(0.02) \mathrm{a}$ & $59(0.66) c-f$ & $182(1.6) b c$ \\
\hline D1 & 9 & $8.0(0.03) \mathrm{bc}$ & $0.19(0.02) \mathrm{ab}$ & $62(0.41)$ ef & $250(12.0) \mathrm{cde}$ \\
\hline D2 & 9 & $7.8(0.05) \mathrm{b}$ & $0.34(0.02) \mathrm{e}$ & $63(0.62) \mathrm{f}$ & 325 (31.8)ef \\
\hline
\end{tabular}

Within each column, means followed by similar letter are not significantly different according to Fisher LSD test $(\mathrm{p}>0.05)$.

in all samples at various treatments and time of sample collections.

Exchangeable cations and CEC varied with treatments and time of sampling (Table 4). In PC1 and CS1 collections, exchangeable Na in D1 and D2 > control while the reverse trend was observed in CS2. Exchangeable K contents $\left(\mathrm{cmol}_{+} \mathrm{kg}^{-1}\right)$ ranged from 0.27 in CS2-Control to 1.60 in PC2-D2. Treatments D1 and D2 contained higher exchangeable $\mathrm{Mg}$ than control plots at all sampling events. There was no clear trend with respect to the influence of PS additions at various times of sampling on changes in the CEC of soils that varied from 7.9 in RES-D2 to 10.7 $\mathrm{cmol}_{+} \mathrm{kg}^{-1}$ in PC1-D2 plots.

The amounts of DTPA-extractable metals showed that DTPA-Zn contents in D2 plots (7.0-18.2 $\mathrm{mg} \mathrm{Zn} \mathrm{kg}^{-1}$ ) at all sampling events were significantly higher than in control samples (0.02-0.51 $\mathrm{mg} \mathrm{Zn} \mathrm{kg}^{-1}$ ) (Table 5). Iron extractable by DTPA in D1 (1.8-7.2 $\left.\mathrm{mg} \mathrm{Fe} \mathrm{kg}^{-1}\right)$ and D2 (5.0-11.3 mg $\mathrm{Fe} \mathrm{kg}{ }^{-1}$ ) plots were higher than control (3.5-6.1 mg Fe kg-1) in samples collected during PC1, CS1, and PC2 events. DTPA-Cu contents (mg Cu kg-1) ranged from 1.1 (CS2Control) to 3.3 (PC1-D2) while contents of Mn in DTPA extracts ( $\mathrm{mg} \mathrm{Mn} \mathrm{kg}^{-1}$ ) varied from 12.2 in CS2-control to 106.0 in PC1-D2 (Table 5).

\section{Biomass yield and uptake of major and minor nutrients by spring barley}

The total biomass of spring barley in D1 and D2 plots were higher compared to control plots in both 2010 and 2011 crop seasons (Figure 1). It was noted that biomass yields 
Table 3. Changes to mean and standard error (parentheses) of $\mathrm{NO}_{3}{ }^{-}-\mathrm{N}, \mathrm{NH}_{4}{ }^{+}-\mathrm{N}$, total $\mathrm{N}$, organic $\mathrm{C}$, and $\mathrm{C} / \mathrm{N}$ ratio at five sampling periods during two consecutive cropping seasons of spring barley grown in agricultural soil amended with two doses $\left(D 1=170 \mathrm{~kg} \mathrm{~N} \mathrm{ha}^{-1} \mathrm{yr}^{-1} ; \mathrm{D} 2=\right.$ $340 \mathrm{~kg} \mathrm{~N} \mathrm{ha}^{-1} \mathrm{yr}^{-1}$ ) and two applications (October 2010 and 2011) of pig slurry in Lorca southeast Spain, $n=$ number of observations.

\begin{tabular}{|c|c|c|c|c|c|c|}
\hline \multirow[b]{2}{*}{ Treatment } & \multirow[b]{2}{*}{$\mathrm{n}$} & $\mathrm{NO}_{3}^{-}-\mathrm{N}$ & $\mathrm{NH}_{4}{ }^{+}-\mathrm{N}$ & Total $\mathrm{N}^{*}$ & Org C & \multirow[b]{2}{*}{$\mathrm{C} / \mathrm{N}$} \\
\hline & & \multicolumn{3}{|c|}{$\mathrm{mg} \mathrm{kg}^{-1} \longrightarrow$} & $\mathrm{g} \mathrm{kg}^{-1}$ & \\
\hline \multicolumn{7}{|c|}{ Pre-crop 1 (November 2009) } \\
\hline Control & 3 & $9.6(0.50) \mathrm{ab}^{\dagger}$ & $20.9(0.38) \mathrm{fg}$ & $555(7)$ & $9.9(1.06) \mathrm{abc}$ & $17(1.6) \mathrm{a}-\mathrm{e}$ \\
\hline D1 & 9 & $9.8(1.41) \mathrm{ab}$ & $22.6(2.42) \mathrm{g}$ & $663(63)$ & $8.3(1.01) \mathrm{a}$ & $12(0.5) \mathrm{a}$ \\
\hline D2 & 9 & $22.6(0.66) \mathrm{b}$ & $22.1(0.15) \mathrm{g}$ & $772(6)$ & $10.3(0.41) \mathrm{ab}$ & $13(0.4) \mathrm{ab}$ \\
\hline \multicolumn{7}{|c|}{ Crop season 1 (March 2010) } \\
\hline Control & 3 & $3.2(0.37) \mathrm{a}$ & $3.4(0.12) \mathrm{a}$ & $427(28)$ & $15.8(0.64)$ efg & $37(3.8) \mathrm{f}$ \\
\hline D1 & 9 & $9.7(1.95) \mathrm{a}$ & $12.9(1.59) \mathrm{d}$ & $650(68)$ & 14.5 (0.73)efg & $24(2.1) \mathrm{e}$ \\
\hline D2 & 9 & $6.8(0.67) \mathrm{a}$ & $14.5(0.88) \mathrm{de}$ & $630(26)$ & $14.2(0.53) \mathrm{d}-\mathrm{g}$ & $23(1.9) \mathrm{e}$ \\
\hline \multicolumn{7}{|c|}{ Residual (July 2010) } \\
\hline Control & 3 & $3.6(0.10) \mathrm{a}$ & $17.8(2.53) \mathrm{ef}$ & $531(7)$ & $13.1(1.50) \mathrm{b}-\mathrm{g}$ & $25(3.1) \mathrm{e}$ \\
\hline D1 & 9 & $7.9(1.35) \mathrm{a}$ & $6.0(0.91) \mathrm{g}$ & $665(45)$ & $15.6(0.93) \mathrm{fg}$ & $24(1.7) \mathrm{e}$ \\
\hline $\mathrm{D} 2$ & 9 & $15.5(0.45) \mathrm{ab}$ & $9.7(0.74) \mathrm{bc}$ & $546(21)$ & $12.1(0.32) \mathrm{bcd}$ & $22(1.4) \mathrm{de}$ \\
\hline \multicolumn{7}{|c|}{ Pre-crop 2 (December 2010) } \\
\hline Control & 3 & $3.9(0.23) \mathrm{a}$ & $12.8(1.27) \mathrm{cde}$ & $559(6)$ & $10.6(0.72) \mathrm{abc}$ & $19(1.1) \mathrm{a}-\mathrm{e}$ \\
\hline D1 & 9 & $78.5(8.53) \mathrm{c}$ & $6.7(0.88) \mathrm{ab}$ & $797(73)$ & $12.0(0.83) \mathrm{bcd}$ & $15(1.8) \mathrm{abc}$ \\
\hline D2 & 9 & $109.0(11.80) \mathrm{d}$ & $15.2(0.48) \mathrm{de}$ & $892(74)$ & $15.5(1.44) \mathrm{fg}$ & $17(2.7) \mathrm{bcd}$ \\
\hline \multicolumn{7}{|c|}{ Crop season 2 (June 2011) } \\
\hline Control & 3 & $3.6(0.56) \mathrm{a}$ & $5.5(0.15) \mathrm{ab}$ & $560(81)$ & $12.5(1.44) \mathrm{b}-\mathrm{f}$ & $22(2.9) \mathrm{de}$ \\
\hline D1 & 9 & $14.6(3.84) \mathrm{ab}$ & $5.9(0.70) \mathrm{a}$ & $673(47)$ & $13.1(0.83) \mathrm{cde}$ & $19(1.5) \mathrm{cde}$ \\
\hline D2 & 9 & $73.6(1.52) \mathrm{c}$ & $7.3(0.73) \mathrm{ab}$ & $748(47)$ & $16.2(0.83) \mathrm{g}$ & $21(2.3) \mathrm{cd}$ \\
\hline
\end{tabular}

Within each column, means followed by similar letter are not significantly different according to Fisher LSD test $(\mathrm{p}>0.05)$.

¥ Non significant.

Table 4. Changes to mean and standard error (parentheses) of exchangeable $\mathrm{Na}, \mathrm{K}, \mathrm{Mg}$, and $\mathrm{CEC}$ at five sampling periods during two consecutive cropping seasons of spring barley grown in agricultural soil amended with two doses $\left(\mathrm{D} 1=170 \mathrm{~kg} \mathrm{~N} \mathrm{ha}^{-1} \mathrm{yr}^{-1}\right.$; D2 $\left.=340 \mathrm{~kg} \mathrm{~N} \mathrm{ha}^{-1} \mathrm{yr}^{-1}\right)$ and two applications (October 2010 and 2011) of pig slurry in Lorca southeast Spain, $n=$ number of observations.

\begin{tabular}{|c|c|c|c|c|c|}
\hline \multirow{2}{*}{ Treatment } & & Exch $\mathrm{Na}$ & Exch $\mathrm{K}$ & Exch Mg & CEC \\
\hline & $\mathrm{n}$ & \multicolumn{4}{|c|}{$\mathrm{cmol}+\mathrm{kg}^{-1}$} \\
\hline \multicolumn{6}{|c|}{ Pre-crop 1 (November 2009) } \\
\hline Control & 3 & $0.86(0.05) \mathrm{def}$ & $0.38(0.08) \mathrm{ab}$ & $0.70(0.02) \mathrm{ab}$ & 9.3 (0.43)de \\
\hline D1 & 9 & $1.10(0$ & $0.83(0.20) \mathrm{bcd}$ & $0.86(0.03) \mathrm{ab}$ & $9.7(0.21) \mathrm{e}$ \\
\hline D2 & 9 & $1.20(0.05) \mathrm{g}$ & $0.37(0.5) \mathrm{a}$ & $0.70(0.02) \mathrm{f}$ & $10.7(0.26) \mathrm{f}$ \\
\hline \multicolumn{6}{|c|}{ Crop season 1 (March 2010) } \\
\hline Control & 3 & $0.16(0.02) \mathrm{a}$ & $0.36(0.05) \mathrm{ab}$ & $0.69(0.01) \mathrm{ab}$ & $9.0(0.02) \mathrm{b}-\mathrm{e}$ \\
\hline D1 & 9 & 0.18 & $1.10(0.29) \mathrm{d}$ & $0.81(0.04) \mathrm{ab}$ & $8.8(0.15) \mathrm{bd}$ \\
\hline $\mathrm{D} 2$ & 9 & $0.50(0.14) \mathrm{bc}$ & $0.79(0.03) \mathrm{bcd}$ & $1.50(0.09) \mathrm{e}$ & $8.1(0.14) \mathrm{ac}$ \\
\hline \multicolumn{6}{|c|}{ Residual (July 2010) } \\
\hline Control & 3 & $0.39(0.06) \mathrm{abc}$ & $0.42(0.02) \mathrm{ab}$ & $0.71(0.02) \mathrm{ab}$ & $8.5(0.07) \mathrm{a}-\mathrm{d}$ \\
\hline D1 & 9 & $0.48(0.07) \mathrm{bc}$ & $1.00(0.22) \mathrm{cd}$ & $0.85(0.04) \mathrm{ab}$ & $8.1(0.29) \mathrm{abc}$ \\
\hline D2 & 9 & $0.62(0.02) \mathrm{cd}$ & $0.77(0.07) \mathrm{a}-\mathrm{e}$ & $1.30(0.04) \mathrm{de}$ & $7.9(0.49) \mathrm{a}$ \\
\hline \multicolumn{6}{|c|}{ Pre-crop 2 (December 2010) } \\
\hline Control & 3 & $0.73(0.09)$ cde & $0.48(0.24) \mathrm{abc}$ & $0.97(0.08) \mathrm{bc}$ & $8.9(0.43) \mathrm{a}-\mathrm{e}$ \\
\hline D1 & 9 & $0.73(0.05) \mathrm{d}$ & $1.00(0.14) \mathrm{cd}$ & $1.20(0.04) \mathrm{cd}$ & $10.6(0.38) \mathrm{f}$ \\
\hline D2 & 9 & $1.00(0.04) \mathrm{efg}$ & $1.60(0.09) \mathrm{e}$ & $1.80(0.08) \mathrm{f}$ & $9.2(0.11) \mathrm{de}$ \\
\hline \multicolumn{6}{|c|}{ Crop season 2 (June 2011) } \\
\hline Control & 3 & $1.20(0.05) \mathrm{g}$ & $0.27(0.01)$ & $0.66(0.02) \mathrm{a}$ & $8.4(0.21) \mathrm{a}-\mathrm{d}$ \\
\hline D1 & 9 & $0.34(0.04) \mathrm{ab}$ & $0.84(0.15) \mathrm{bcd}$ & $0.79(0.03) \mathrm{a}$ & $8.9(0.29) \mathrm{bd}$ \\
\hline D2 & 9 & $0.64(0.07) \mathrm{cd}$ & $1.10(0.03) \mathrm{d}$ & $1.20(0.01) \mathrm{d}$ & $8.0(0.07) \mathrm{ac}$ \\
\hline
\end{tabular}

Within each column, means followed by similar letter are not significantly different according to Fisher LSD test $(\mathrm{p}>0.05)$.

CEC: cation exchange capacity.

in $2011\left(323-663 \mathrm{~kg} \mathrm{ha}^{-1}\right)$ were almost half compared to $2010\left(799-1342 \mathrm{~kg} \mathrm{ha}^{-1}\right)$. Contents of available $\mathrm{NO}_{3}^{-}-\mathrm{N}$ in PC1 and RES were significantly correlated with the 2010 and 2011 yields with $\mathrm{R}$ values equal to 0.86 and 0.85 , respectively (Figure 1, Table 7). Olsen-P were also highly correlated with biomass yields $(\mathrm{R}=0.90$ for both 2010 and 2011). Among minor elements, PC1- and RESDTPA-Zn had the highest correlations with yields at $\mathrm{R}=$
0.83 and 0.90 for 2010 and 2011, respectively (Table 6).

Total uptake of spring barley for various nutrients correlated well with soil variables measured in the study (Figures 2 and 3, Table 7). Total $\mathrm{N}$ uptake $\left(\mathrm{mg} \mathrm{kg}^{-1}\right)$ of barley ranged from low $<2000$ (2010 grain) to about 24 500 (2010 grain and ear). In 2010 , total $\mathrm{N}$ in plants was best correlated with available $\mathrm{NO}_{3}^{-}-\mathrm{N}$ in $\mathrm{PC} 1(\mathrm{R}=0.58$ and 0.71 , respectively for grain and ear) while available $\mathrm{NO}_{3}-\mathrm{N}$ in $\mathrm{CS} 2$ had the highest $\mathrm{R}$ values $(0.83$ and 0.79 ,

Table 5. Changes to mean and standard error (parentheses) of bioavailable $\mathrm{Zn}, \mathrm{Fe}, \mathrm{Cu}$, and $\mathrm{Mn}$ at five sampling periods during two consecutive cropping seasons of spring barley grown in agricultural soil amended with two doses $\left(\mathrm{D} 1=170 \mathrm{~kg} \mathrm{~N} \mathrm{ha}^{-1} \mathrm{yr}^{-1} ; \mathrm{D} 2=340 \mathrm{~kg} \mathrm{~N}\right.$ $\mathrm{ha}^{-1} \mathrm{yr}^{-1}$ ) and two applications (October 2010 and 2011) of pig slurry in Lorca southeast Spain, $n=$ number of observations.

\begin{tabular}{|c|c|c|c|c|c|}
\hline \multirow[b]{2}{*}{ Treatment } & \multirow[b]{2}{*}{$\mathrm{n}$} & $\mathrm{Zn}$ & $\mathrm{Fe}$ & $\mathrm{Cu}$ & $\mathrm{Mn}$ \\
\hline & & \multicolumn{4}{|c|}{$\mathrm{mg} \mathrm{kg}^{-1}$} \\
\hline \multicolumn{6}{|c|}{ Pre-crop 1 (November 2009) } \\
\hline Control & 3 & $0.49(0.09) \mathrm{ab}^{\dagger}$ & $6.1(0.39)$ ef & $2.0(0.21) \mathrm{b}$ & $42.2(2.5) \mathrm{e}$ \\
\hline D1 & 9 & $2.60(1.02) \mathrm{ab}$ & $7.2(0.74) \mathrm{f}$ & $2.6(0.15) \mathrm{cd}$ & $68.2(9.45) \mathrm{f}$ \\
\hline D2 & 9 & $18.20(1.70) \mathrm{d}$ & $11.3(0.58) \mathrm{g}$ & $3.3(0.13) \mathrm{e}$ & $106.0(4.24) \mathrm{g}$ \\
\hline \multicolumn{6}{|c|}{ Crop season 1 (March 2010) } \\
\hline Control & 3 & $0.51(0.02) \mathrm{ab}$ & $3.5(0.34) \mathrm{bc}$ & $2.0(0.09) \mathrm{b}$ & $15.4(0.56) \mathrm{abc}$ \\
\hline D1 & 9 & $2.70(1.01) \mathrm{ab}$ & $3.8(0.23) \mathrm{c}$ & $2.4(0.14) \mathrm{bc}$ & $20.8(1.71) \mathrm{abc}$ \\
\hline D2 & 9 & $7.70(0.58) \mathrm{c}$ & $5.0(0.33) \mathrm{de}$ & $2.4(0.10) \mathrm{bc}$ & 24.9 (1.14)bcd \\
\hline \multicolumn{6}{|c|}{ Residual (July 2010) } \\
\hline Control & 3 & $0.32(0.03) \mathrm{a}$ & $3.7(0.03) \mathrm{cd}$ & $2.7(0.15) \mathrm{cd}$ & $16.7(0.38) \mathrm{abc}$ \\
\hline D1 & 9 & $2.80(1.16) \mathrm{ab}$ & $3.7(0.07) \mathrm{cd}$ & $2.5(0.12) \mathrm{cd}$ & $20.8(1.02) \mathrm{abc}$ \\
\hline D2 & 9 & $7.30(0.46) \mathrm{c}$ & $4.4(0.11) \mathrm{cd}$ & $2.1(0.06) \mathrm{b}$ & $19.2(0.81) \mathrm{abc}$ \\
\hline \multicolumn{6}{|c|}{ Pre-crop 2 (December 2010) } \\
\hline Control & 3 & $0.36(0.06) \mathrm{a}$ & 4.7 (0.26)cde & $2.0(0.22) \mathrm{b}$ & 25.8 (3.71)abcd \\
\hline D1 & 9 & $3.90(0.73) \mathrm{b}$ & $4.6(0.21) \mathrm{cd}$ & $2.3(0.12) \mathrm{bc}$ & $27.6(1.59) \mathrm{cd}$ \\
\hline D2 & 9 & $16.2(0.89) \mathrm{d}$ & $6.8(0.38) \mathrm{f}$ & $2.8(0.08) \mathrm{d}$ & $32.8(0.62) \mathrm{de}$ \\
\hline \multicolumn{6}{|c|}{ Crop season 2 (June 2011) } \\
\hline Control & 3 & $0.02(0.003) \mathrm{a}$ & $1.7(0.02) \mathrm{a}$ & $1.1(0.04) \mathrm{a}$ & $12.2(0.83) \mathrm{ab}$ \\
\hline D1 & 9 & $2.20(0.73) \mathrm{a}$ & $1.8(0.23) \mathrm{a}$ & $1.2(0.09) \mathrm{a}$ & $14.8(0.65) \mathrm{a}$ \\
\hline D2 & 9 & $7.00(0.51) \mathrm{c}$ & $2.2(0.21) \mathrm{ab}$ & $1.3(0.10) \mathrm{a}$ & $16.0(0.95) \mathrm{ab}$ \\
\hline
\end{tabular}

${ }^{\dagger}$ Within each column, means followed by similar letter are not significantly different according to Fisher LSD test $(p>0.05)$. 
Table 6. Statistically significant correlations between major $(\mathbf{N}$, $\mathrm{P}, \mathrm{K}, \mathrm{Mg}$ ) and minor ( $\mathrm{Zn}, \mathrm{Fe}, \mathrm{Cu}$, and $\mathrm{Mn}$ ) and biomass of spring barley grown in 2010 and 2011 in an agricultural soil amended with two doses (D1 $\left.=170 \mathrm{~kg} \mathrm{~N}^{-1} \mathrm{yr}^{-1} ; \mathrm{D} 2=340 \mathrm{~kg} \mathrm{~N}^{-1} \mathrm{yr}^{-1}\right)$ and two applications (October 2010 and 2011) of pig slurry in Lorca southeast Spain.

\begin{tabular}{lcccc}
\hline & 2010 & 2011 & 2011 & 2011 \\
& Pre-Crop 1 & Residual & Pre-Crop 2 & Crop Season 2 \\
\hline Available $\mathrm{NO}_{3}-\mathrm{N}$ & 0.86 & 0.85 & $\mathrm{~ns}$ & $\mathrm{~ns}$ \\
Available $\mathrm{NH}_{4}{ }^{+}-\mathrm{N}$ & $\mathrm{ns}$ & $\mathrm{ns}$ & $\mathrm{ns}$ & $\mathrm{ns}$ \\
Total N & 0.85 & $\mathrm{~ns}$ & 0.90 & 0.87 \\
Organic N & 0.84 & $\mathrm{~ns}$ & 0.90 & 0.86 \\
Olsen-P & 0.86 & 0.90 & $\mathrm{~ns}$ & 0.82 \\
Exchangeable K & $\mathrm{ns}$ & $\mathrm{ns}$ & 0.83 & 0.78 \\
Exchangeable $\mathrm{Mg}$ & 0.79 & 0.78 & $\mathrm{~ns}$ & $\mathrm{~ns}$ \\
DTPA-Zn & 0.83 & 0.90 & $\mathrm{~ns}$ & 0.86 \\
DTPA-Fe & 0.83 & $\mathrm{~ns}$ & $\mathrm{~ns}$ & 0.79 \\
DTPA-Cu & 0.87 & $\mathrm{~ns}$ & $\mathrm{~ns}$ & $\mathrm{~ns}$ \\
DTPA-Mn & 0.90 & $\mathrm{~ns}$ & $\mathrm{~ns}$ & 0.83 \\
\hline
\end{tabular}

DTPA: diethylene-triamine pentaacetic acid.

Table 7. Statistically significant correlations between available $\mathrm{NO}_{3}$. $-\mathrm{N}, \mathrm{NH}_{4}{ }^{+}-\mathrm{N}$, total $\mathbf{N}$, Olsen-P, exchangeable $\mathrm{K}, \mathrm{Mg}, \mathrm{Na}, \mathrm{DTPA}-\mathrm{Zn}$, $-\mathrm{Fe},-\mathrm{Cu}$ and $-\mathrm{Mn}$, and uptake of $\mathrm{N}, \mathrm{P}, \mathrm{K}, \mathrm{Mg}, \mathrm{Na}, \mathrm{Zn}, \mathrm{Fe}, \mathrm{Cu}$, and $\mathrm{Mn}$ in grains and ear of spring barley grown in 2010 and 2011 in an agricultural soil amended with two doses $\left(\mathrm{D} 1=170 \mathrm{~kg} \mathrm{~N} \mathrm{ha}^{-1} \mathrm{yr}^{-1} ; \mathrm{D} 2\right.$ $=340 \mathrm{~kg} \mathrm{~N} \mathrm{ha}^{-1} \mathrm{yr}^{-1}$ ) and two applications (October 2010 and 2011) of pig slurry in Lorca southeast Spain.

\begin{tabular}{|c|c|c|c|c|c|}
\hline Crop Season 2010 & $\begin{array}{l}2010 \\
\text { Grain }\end{array}$ & $\begin{array}{c}2010 \\
\text { Ear }\end{array}$ & Crop Season 2011 & $\begin{array}{l}2011 \\
\text { Grain }\end{array}$ & $\begin{array}{c}2010 \\
\text { Ear }\end{array}$ \\
\hline & & & \multicolumn{3}{|l|}{ Residual } \\
\hline & & & $\mathrm{NO}_{3}-\mathrm{N}$ & 0.80 & 0.83 \\
\hline & & & $\mathrm{NH}_{4}{ }^{+}-\mathrm{N}$ & $\mathrm{ns}$ & ns \\
\hline & & & Total N & $\mathrm{ns}$ & ns \\
\hline & & & Olsen-P & ns & ns \\
\hline & & & Exchangeable $\mathrm{K}$ & ns & 0.66 \\
\hline & & & Exchangeable $\mathrm{Mg}$ & 0.63 & 0.66 \\
\hline & & & Exchangeable $\mathrm{Na}$ & 0.57 & 0.58 \\
\hline & & & DTPA-Zn & 0.84 & 0.86 \\
\hline & & & DTPA-Fe & 0.56 & $\mathrm{~ns}$ \\
\hline & & & DTPA-Cu & ns & ns \\
\hline & & & DTPA-Mn & ns & ns \\
\hline \multicolumn{3}{|l|}{ Pre-Crop 1} & \multicolumn{3}{|l|}{ Pre-Crop 2} \\
\hline$\overline{\mathrm{NO}_{3}{ }^{\circ}-\mathrm{N}}$ & 0.58 & 0.71 & $\mathrm{NO}_{3}-\mathrm{N}$ & ns & ns \\
\hline $\mathrm{NH}_{4}{ }^{+}-\mathrm{N}$ & $\mathrm{ns}$ & $\mathrm{ns}$ & $\mathrm{NH}_{4}{ }^{+}-\mathrm{N}$ & 0.78 & 0.75 \\
\hline Total N & ns & ns & Total N & 0.43 & 0.56 \\
\hline Olsen-P & 0.59 & 0.66 & Olsen-P & 0.49 & 0.48 \\
\hline Exchangeable $\mathrm{K}$ & -0.56 & -0.45 & Exchangeable $\mathrm{K}$ & 0.55 & 0.62 \\
\hline Exchangeable $\mathrm{Mg}$ & 0.57 & 0.67 & Exchangeable $\mathrm{Mg}$ & 0.58 & 0.60 \\
\hline Exchangeable $\mathrm{Na}$ & $\mathrm{ns}$ & ns & Exchangeable Na & 0.56 & 0.48 \\
\hline DTPA-Zn & 0.73 & 0.74 & DTPA-Zn & 0.94 & 0.93 \\
\hline DTPA-Fe & 0.45 & 0.65 & DTPA-Fe & 0.49 & ns \\
\hline DTPA-Cu & $\mathrm{ns}$ & ns & DTPA-Cu & 0.51 & 0.60 \\
\hline DTPA-Mn & ns & 0.62 & DTPA-Mn & 0.64 & 0.60 \\
\hline \multicolumn{3}{|l|}{ Crop season 1} & \multicolumn{3}{|l|}{ Crop season 2} \\
\hline $\mathrm{NO}_{3}-\mathrm{N}$ & $\mathrm{ns}$ & ns & $\mathrm{NO}_{3}^{-}-\mathrm{N}$ & 0.83 & 0.79 \\
\hline $\mathrm{NH}_{4}^{+}-\mathrm{N}$ & ns & ns & $\mathrm{NH}_{4}{ }^{+}-\mathrm{N}$ & ns & $\mathrm{ns}$ \\
\hline Total N & ns & ns & Total N & 0.47 & 0.52 \\
\hline Olsen-P & ns & ns & Olsen-P & 0.43 & 0.47 \\
\hline Exchangeable $\mathrm{K}$ & ns & ns & Exchangeable $\mathrm{K}$ & 0.65 & 0.83 \\
\hline Exchangeable Mg & 0.61 & 0.66 & Exchangeable $\mathrm{Mg}$ & 0.68 & 0.70 \\
\hline Exchangeable $\mathrm{Na}$ & ns & ns & Exchangeable $\mathrm{Na}$ & $\mathrm{ns}$ & $\mathrm{ns}$ \\
\hline DTPA-Zn & 0.79 & 0.79 & DTPA-Zn & 0.91 & 0.91 \\
\hline DTPA-Fe & $\mathrm{ns}$ & 0.68 & DTPA-Fe & ns & ns \\
\hline DTPA-Cu & ns & ns & DTPA-Cu & ns & 0.49 \\
\hline DTPA-Mn & 0.65 & 0.57 & DTPA-Mn & 0.43 & 0.46 \\
\hline
\end{tabular}

DTPA: diethylene-triamine pentaacetic acid.

respectively for grain and ear) for the 2011 season (Figure 2A). Other correlations between soil $\mathrm{N}$ and plant $\mathrm{N}$ contents were summarized in Table 7. Correlations between Olsen-P (available P), exchangeable $\mathrm{K}$ and $\mathrm{Mg}$ and total $\mathrm{P}, \mathrm{K}$, and $\mathrm{Mg}$ contents in plants were best shown in $\mathrm{PC} 1$ and CS2 (Figure 2B) and CS1 and CS2 (Figures $2 \mathrm{C}$ and $2 \mathrm{D}$ ) while Table 7 reported the relationships at other sampling periods. Total $\mathrm{P}$ in barley $\left(\mathrm{mg} \mathrm{P} \mathrm{kg}{ }^{-1}\right)$ ranged from 2950 to 6370 (Figure 2B) while total $\mathrm{K}$ in plants $\left(\mathrm{mg} \mathrm{K} \mathrm{kg}^{-1}\right)$ varied between 3681 in 2010 grain to 8000 in 2011 ear (Figure 2C) and for total Mg in plants, 769 and $1528 \mathrm{mg} \mathrm{Mg} \mathrm{kg}^{-1}$ were the lower and upper limits, respectively (Figure 2D).

The uptake of minor nutrients (i.e., $\mathrm{Zn}, \mathrm{Fe}, \mathrm{Cu}$, and $\mathrm{Mn}$ ) and its correlations with soil metals were summarized in Figure 3 and Table 7. Total $\mathrm{Zn}$ content in barley ranged from 15 to $64 \mathrm{mg} \mathrm{Zn} \mathrm{kg}^{-1}$ (2011 ear); total Fe uptake varied from $15 \mathrm{mg} \mathrm{Fe} \mathrm{kg}^{-1}$ (2010 grain) to $53 \mathrm{mg} \mathrm{Fe} \mathrm{kg}{ }^{-1}$ (2011 ear) while the changes to the total $\mathrm{Cu}$ uptake were from $1.8 \mathrm{mg} \mathrm{Cu} \mathrm{kg}^{-1}$ (2010 ear) to $11 \mathrm{mg} \mathrm{Cu} \mathrm{kg}^{-1}$ in 2011 ear, and the total Mn content in barley had lowest value at $13 \mathrm{mg} \mathrm{Mn} \mathrm{kg}{ }^{-1}$ in 2010 grain and highest value at $35 \mathrm{mg}$ $\mathrm{Mn} \mathrm{kg}^{-1}$ in 2011 ear. The best correlations between total uptake of minor elements and DTPA-minor elements in soil were in soil samples collected in PC1 (2010) and PC2 (2011) (Figure 3 and Table 7).

\section{DISCUSSION}

\section{Changes to soil properties with PS additions}

The limited changes to soil $\mathrm{pH}$ after PS applications could be related to the high $\mathrm{CaCO}_{3}$-based buffer capacity of the soil $\left(52-68 \% \mathrm{CaCO}_{3}\right)$ as well as to the relatively high $\mathrm{pH}$ (7.2 and 7.6) of PS. Reduction to $\mathrm{pH} 7.4$ observed in PC2-D2 involved the dissolution of some $\mathrm{CaCO}_{3}$ originally present in the soil although not apparent change in $\mathrm{CaCO}_{3}$ contents was detected in the study. The high $\mathrm{CaCO}_{3}$ content provides high amounts of $\mathrm{Ca}^{2+}$ to maintain the ionic strength of the soil thus, prevent any dramatic increase or decrease in EC, all EC values are all below the $4 \mathrm{dS} \mathrm{m}^{-1}$ limit for salt-affected soils (Richards, 1954).

The addition of PS has been reported to improve the $\mathrm{N}$ status of soils for agricultural or environmental purposes (Chantigny et al., 2001; Daudén and Quílez, 2004; Whalen and DeBerardinis, 2007; Cela et al., 2011). Although the total $\mathrm{N}$ in the PS (1.00-1.41 $\mathrm{g}$ total $\left.\mathrm{M} \mathrm{kg}^{-1}\right)$ is lower than Chantigny et al. (2001) at $86 \mathrm{~g}$ total $\mathrm{N} \mathrm{kg}^{-1}$, our results are still consistent with the literature because at all stages of sampling, D1 and D2 plots have higher total $\mathrm{N}$ than control due to PS additions. The increase in soil total $\mathrm{N} 1 \mathrm{yr}$ after PS application has been reported in soils planted with corn in northeastern Spain (Cela et al., 2011). Two-time additions of PS to soils in northwest Germany resulted to $70 \mathrm{~kg} \mathrm{~N} \mathrm{ha}^{-1} \mathrm{yr}^{-1} \mathrm{~N}$ surplus compared to negative $\mathrm{N}$ balance in plots without PS addition (Sieling et al., 2006). Contents of $\mathrm{NO}_{3}^{-}-\mathrm{N}$ in PC2-D2 and CS2-D2 are 7X (109 $\mathrm{mg} \mathrm{NO}_{3}{ }^{-} \mathrm{N} \mathrm{kg}^{-1}$ vs. $\left.15.2 \mathrm{mg} \mathrm{NH}_{4}{ }^{+}-\mathrm{N} \mathrm{kg}^{-1}\right)$ and $10 \mathrm{X}(73.6$ mg $\mathrm{NO}_{3}^{-}-\mathrm{N} \mathrm{kg}^{-1}$ vs. $\left.7.3 \mathrm{mg} \mathrm{NH}_{4}{ }^{+} \mathrm{N} \mathrm{kg}^{-1}\right)$, respectively compared to $\mathrm{NH}_{4}{ }^{+} \mathrm{N}$ in the same sampling events. The higher amounts of $\mathrm{NO}_{3}{ }^{-}-\mathrm{N}$ compared to $\mathrm{NH}_{4}{ }^{+}-\mathrm{N}$ after two 

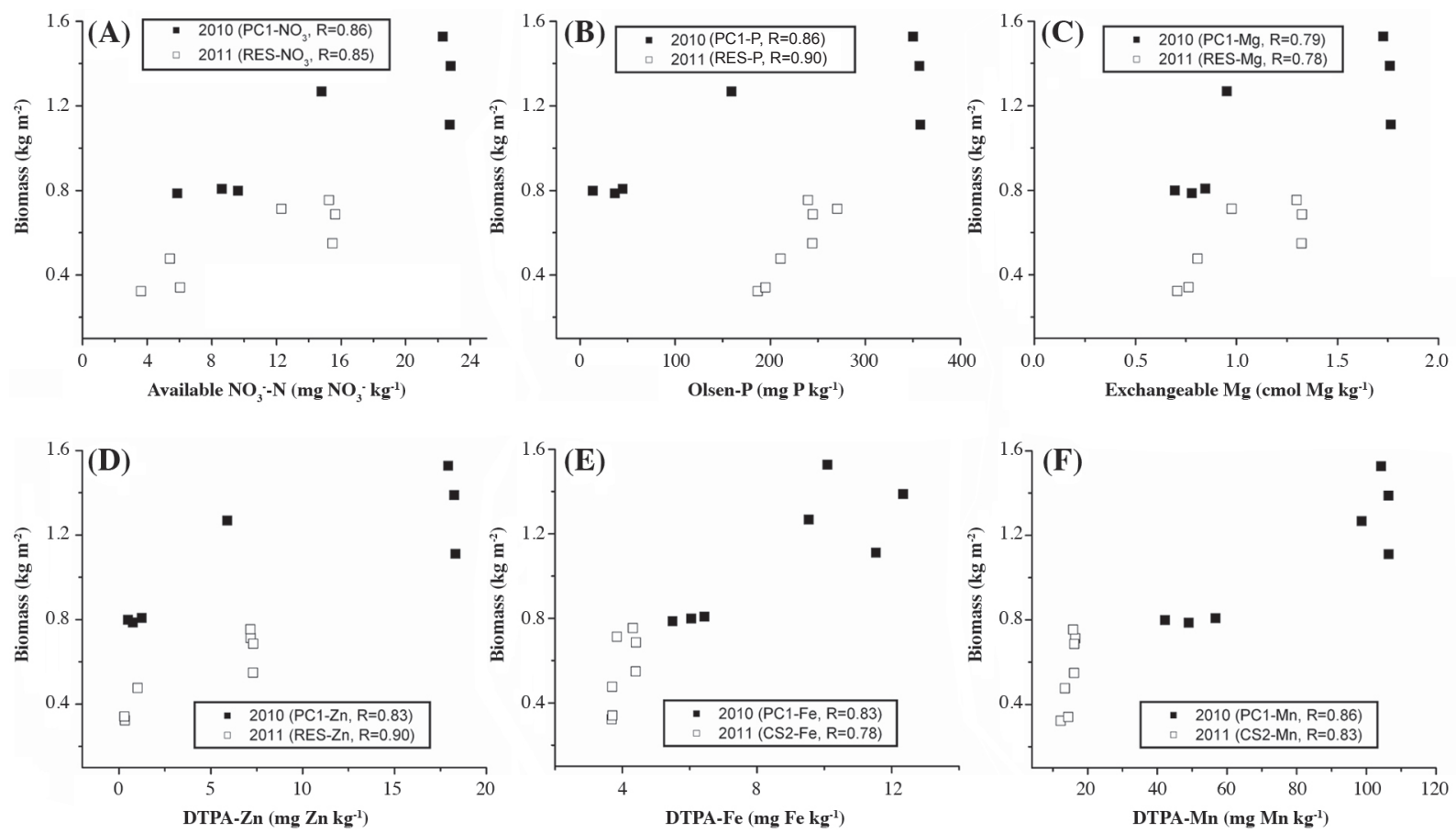

DTPA: diethylene-triamine pentaacetic acid. PC1: Pre-Crop 1; RES: Residual; CS2: Crop Season 2.

Figure 1. Relationships between biomass in 2010 and 2011 and selected soil properties in an agricultural soil amended with two doses (D1 = 170 $\mathrm{kg} \mathrm{N} \mathrm{ha}^{-1} \mathrm{yr}^{-1}$; D2 = $340 \mathrm{~kg} \mathrm{~N} \mathrm{ha}^{-1} \mathrm{yr}^{-1}$ ) and two applications (October 2010 and 2011) of pig slurry in Lorca southeast Spain.

time additions of PS in the study site is consistent with nitrification of $\mathrm{NH}_{4}{ }^{+}-\mathrm{N}$ in PS added to soils in Canada (Chantigny et al., 2001; Whalen and DeBerardinis, 2007).

As expected, available $\mathrm{P}$ (Olsen-P) in soils increased to at least $2 \mathrm{X}$ compared to the control due to high $\mathrm{P}$ content (118 and $184 \mathrm{mg}$ total $\mathrm{PL}^{-1}$ in 2009 and 2010, respectively) in PS materials used in the study. This result is consistent with those of Bernal et al. (1993), who reported increases in available $\mathrm{P}$ after additions of 300 to $400 \mathrm{~m}^{3} \mathrm{PS} \mathrm{ha}^{-1}$ in a greenhouse study using pepper as test plant in SE Spain. Olsen- $\mathrm{P}$ is regarded as the available form of $\mathrm{P}$ especially in soils with high $\mathrm{pH}$ (Olsen et al., 1954) such as in the study area. The lower Olsen-P in RES-D1 and RES-D2 compared to CS1-D1 and CS1-D2 might be attributed to plant uptake of $\mathrm{P}$ or to $\mathrm{P}$-fixation of added $\mathrm{P}$ from PS into non-available form that could be as high as $41 \%$ to $71 \%$ of added P (Bernal et al., 1993). Soils at high pH ( pH 7.0) fix added $\mathrm{P}$ by the reaction of $\mathrm{Ca}$ with $\mathrm{P}$ to form insoluble Ca-P minerals.

The increases in amounts of exchangeable $\mathrm{K}$ and $\mathrm{Mg}$ in soils are due to high $\mathrm{K}\left(836-1020 \mathrm{mg} \mathrm{K} \mathrm{L}^{-1}\right)$ and $\mathrm{Mg}$ (71-145 $\left.\mathrm{mg} \mathrm{Mg} \mathrm{L}^{-1}\right)$ contained in PS used in the experiment. It seems that PS can be an effective source of essential major cations as the amounts of exchangeable $\mathrm{K}$ and $\mathrm{Mg}$ are significantly higher than control plots at several sampling events in the experiment. However, net increases in exchangeable cations from PS additions are dependent on initial CEC of soils (Bernal et al., 1993).
Similarly, $\mathrm{Zn}$ and Fe contained in the PS is likely the cause of the observed increased amounts of these metals extractable in DTPA after two time additions of PS in soils. The amounts of $\mathrm{Cu}\left(0.50-0.67 \mathrm{mg} \mathrm{Cu} \mathrm{L}^{-1}\right)$ and $\mathrm{Mn}$ (0.42-0.69 $\left.\mathrm{mg} \mathrm{Mn} \mathrm{L}^{-1}\right)$ in PS are too low to significantly increased DTPA-Cu and $\mathrm{Mn}$ in soils. In contrast, the increases in $\mathrm{Mn}$ and $\mathrm{Cu}$ contents in soils reported by Bernal et al. (1993) is attributed to high Mn (18-22 mg $\mathrm{Mn} \mathrm{kg}{ }^{-1}$ ) and $\mathrm{Cu}\left(43-48 \mathrm{mg} \mathrm{Cu} \mathrm{kg}{ }^{-1}\right)$ contents in PS amendments.

\section{Yield and uptake of major and minor nutrients with PS additions}

The amount of available $\mathrm{NO}_{3}{ }^{-}-\mathrm{N}$ seems to be the best measure of soil $\mathrm{N}$ with respect to biomass yield and total $\mathrm{N}$ uptake in both ear and grain. In addition, total $\mathrm{N}$, organic $\mathrm{N}$, and available $\mathrm{NO}_{3}{ }^{-}-\mathrm{N}$ (PC1) measured 1-mo after PS applications (PC1 and PC2) are ideal measures (due to high correlation coefficient) to assess the amounts of soil $\mathrm{N}$ related to total biomass of barley in this study. In addition, barley seems to prefer $\mathrm{NO}_{3}{ }^{-} \mathrm{N}$ than $\mathrm{NH}_{4}{ }^{+}-\mathrm{N}$ as source of $\mathrm{N}$ and is in agreement with the earlier findings of Nielsen and Jensen (1986) that preferential absorption of $\mathrm{NO}_{3}{ }^{-}-\mathrm{N}$ over $\mathrm{NH}_{4}{ }^{+}-\mathrm{N}$ occurred at all stages of barley growth.

The highest correlation coefficients between PC1Olsen-P (2010 barley crop) and RES-Olsen-P (2011 barley crop) indicate the sensitivity of barley to available 

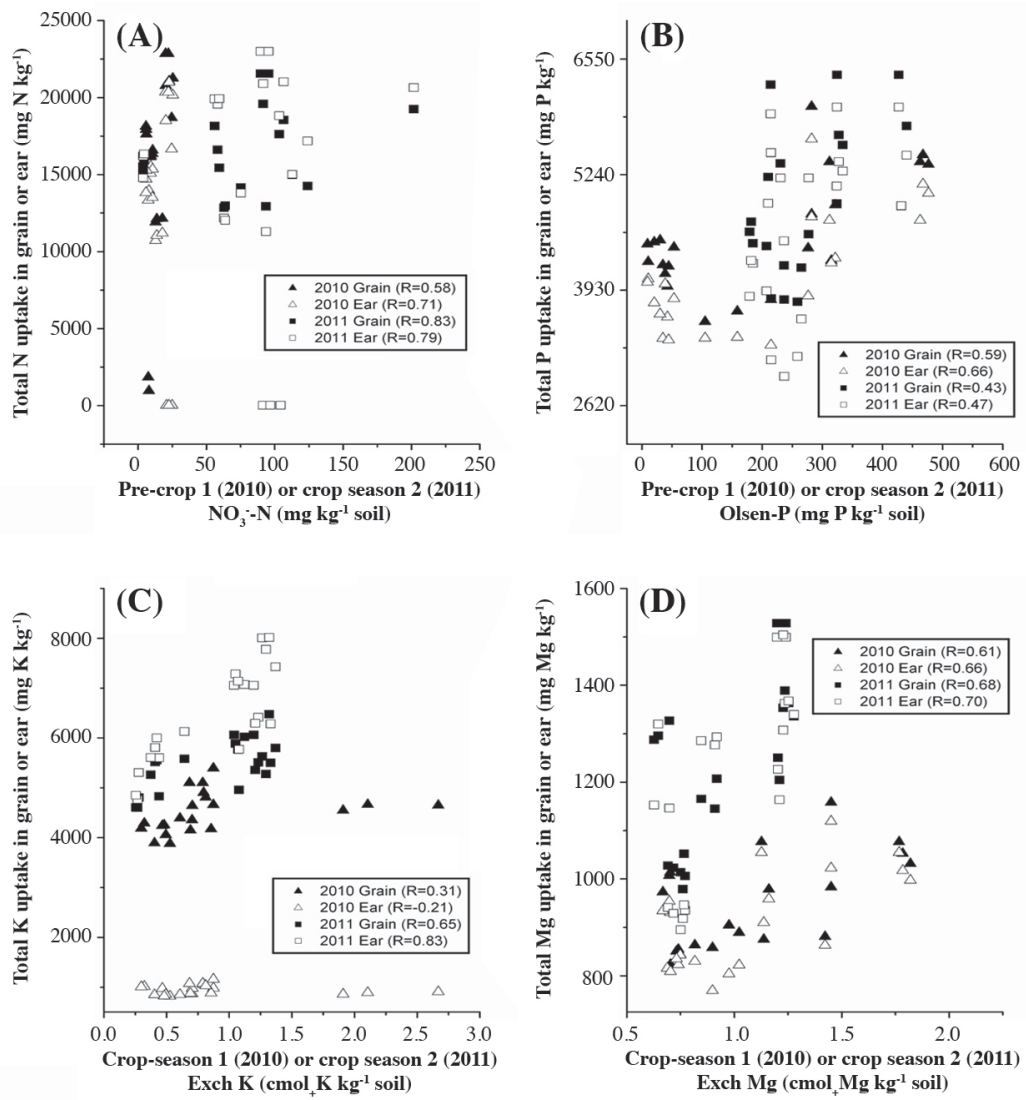

Figure 2. Relationships between nutrient uptake in barley in 2010 and 2011 and selected soil properties in an agricultural soil amended with two doses $\left(\mathrm{D} 1=170 \mathrm{~kg} \mathrm{~N} \mathrm{ha}^{-1} \mathrm{yr}^{-1}\right.$; D2 = $\left.340 \mathrm{~kg} \mathrm{~N} \mathrm{ha}^{-1} \mathrm{yr}^{-1}\right)$ and two applications (October 2010 and 2011) of pig slurry in Lorca, Spain.

$\mathrm{P}$ at the early stage of growth. Our results are in agreement with those of Kuligowski et al. (2010), who reported $100 \%$ higher biomass in spring barley treated with 30 $\mathrm{kg} \mathrm{P} \mathrm{ha-1} \mathrm{using} \mathrm{various} \mathrm{forms} \mathrm{of} \mathrm{fertilizer} \mathrm{derived} \mathrm{from}$ pig manure applied prior to sowing of barley seeds. The amount of available $\mathrm{P}$ is critical at the early stage and may have significant influence on the final biomass yield (Kuligowski et al., 2010). Kuligowski et al. (2010) also reported that $\mathrm{P}$ in liquid fertilizer is more readily available than in dry form. Range of $\mathrm{P}$ uptake in ear and grain in this study (2.6-6.3 $\mathrm{g} \mathrm{P} \mathrm{kg}^{-1}$ biomass) is slightly higher than the 1 and $3.5 \mathrm{~g} \mathrm{P} \mathrm{kg}^{-1}$ biomass reported in literature (Soon, 1988; Pan and Hopkins, 1991; Arvidsson, 1999; Kuligowski et al., 2010).

The inconsistent significant correlations between exchangeable $\mathrm{K}$ and biomass and $\mathrm{K}$ uptake might be due to complex chemistry of $\mathrm{K}$ availability in soils. For example, the availability of $\mathrm{K}$ to plants is best expressed through $\mathrm{K}$-intensity $\left[\mathrm{K}^{+} /\left(\mathrm{Ca}^{2+}+\mathrm{Mg}^{2+}\right)\right]$ because absorption of $\mathrm{K}$ (and $\mathrm{Ca}$ and $\mathrm{Mg}$ ) by plants is governed by the activities of $\mathrm{K}^{+}, \mathrm{Ca}^{2+}$, and $\mathrm{Mg}^{2+}$ rather than individual contents of exchangeable cations (Nielsen and Hansen, 1984). Potassium and $\mathrm{Mg}$ (and other cations such as $\mathrm{Ca}$ ) in soils are positively charged, thus their mobility are limited due potential absorption in negatively-charged exchange sites in soils. Sources of major cations $(\mathrm{K}, \mathrm{Mg}, \mathrm{Ca}$, and $\mathrm{Na}$ ) must be accessible to plant roots for efficient uptake at critical stages of growth to achieve optimal biomass yield (Gardiner and Miller, 2001). The high $\mathrm{Ca}^{2+}$ from the dissolution of $\mathrm{CaCO}_{3}$ precluded us from assessing the status of exchangeable $\mathrm{Ca}^{2+}$ in the study area. It is almost impossible to distinguish exchangeable $\mathrm{Ca}^{2+}$ from those liberated by the dissolution of $\mathrm{CaCO}_{3}$. However, it was found that exchangeable $\mathrm{Mg}$ measured at PC1 and RES is correlated well with biomass and $\mathrm{Mg}$ uptake in ear and grain in both 2010 and 2011 barley crop seasons. In addition, the contents of $\mathrm{K}\left(3.9-8.0 \mathrm{~g} \mathrm{~K} \mathrm{~kg}^{-1}\right)$ and $\mathrm{Mg}$ (0.76-1.5 $\left.\mathrm{g} \mathrm{Mg} \mathrm{kg}^{-1}\right)$ in barley in this study fall within the reported $\mathrm{K}$ (4.9-5.1 $\mathrm{g} \mathrm{kg}^{-1}$ ) and $\mathrm{Mg}$ (1.3-1.4 $\mathrm{g} \mathrm{kg}^{-}$ $\left.{ }^{1}\right)$ contents in literature (Dick et al., 1985; Soon, 1988; Arvidsson, 1999).

For the 2010 barley crop season, DTPA-extractable minor nutrients $(\mathrm{Zn}, \mathrm{Fe}, \mathrm{Cu}$, and $\mathrm{Mn}$ ) at $\mathrm{PC} 1$ may indicate the sensitivity of barley at the very early stage of growth to obtain optimum yield and uptake of minor elements. Obrador et al. (2007) reported a significant relationship $\left(\mathrm{R}^{2}=46 \%\right)$ between DTPA-Zn and Zn uptake by barley but no significant correlation between DTPA-Mn and Mn content in barley. The additions of PS elevated the DTPA-extractable $\mathrm{Zn}$ from $<0.5 \mathrm{mg}$ DTPA-Zn kg-1 soil 

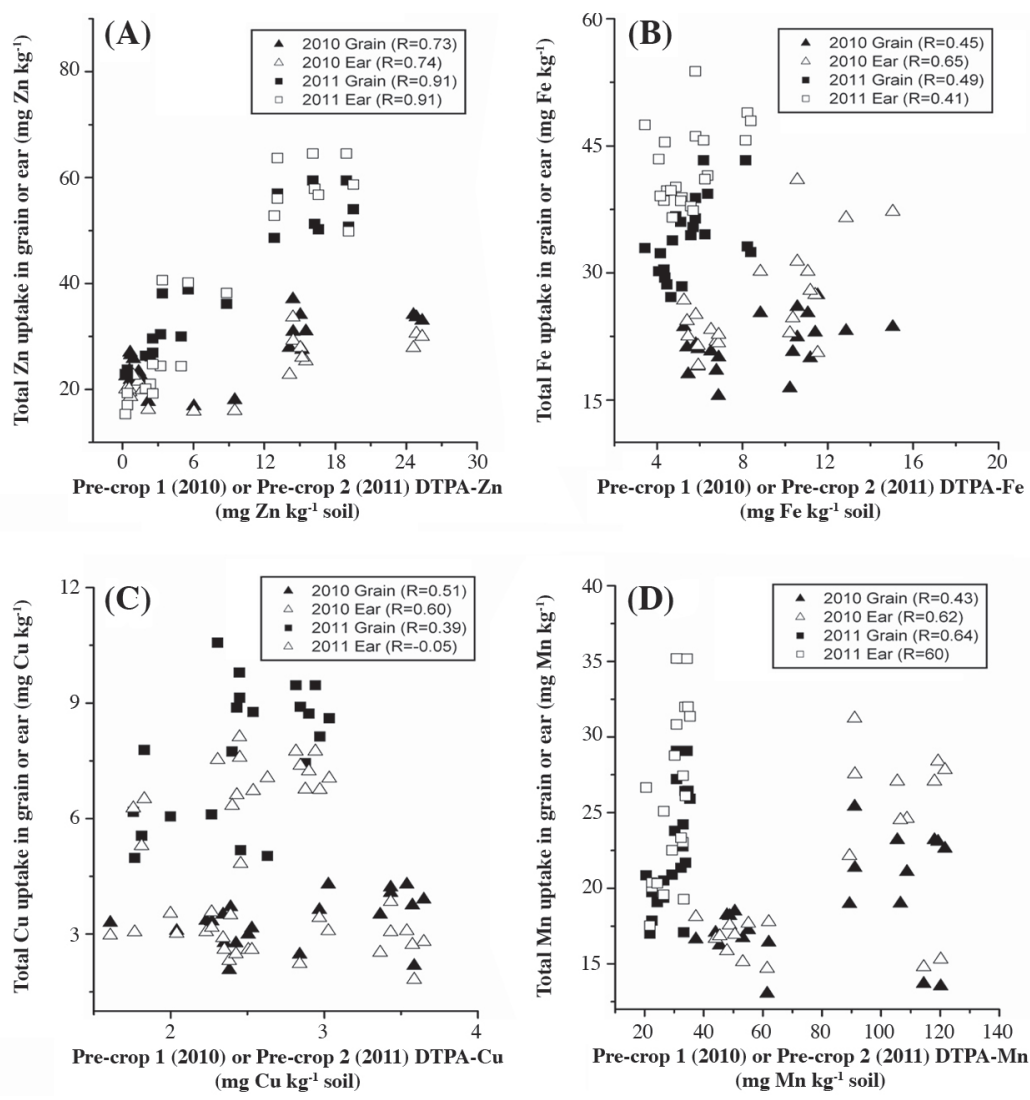

DTPA: diethylene-triamine pentaacetic acid.

Figure 3. Relationships between nutrient uptake in barley in 2010 and 2011 and selected soil properties in an agricultural soil amended with two doses $\left(\mathrm{D} 1=170 \mathrm{~kg} \mathrm{~N} \mathrm{ha}^{-1} \mathrm{yr}^{-1} ; \mathrm{D} 2=340 \mathrm{~kg} \mathrm{~N} \mathrm{ha}^{-1} \mathrm{yr}^{-1}\right)$ and two applications (October 2010 and 2011) of pig slurry in Lorca, Spain.

to above the critical level $~ 0.7 \mathrm{mg}$ DTPA-Zn kg-1 for normal growth of sorghum (Lindsay and Norvell, 1978). However, DTPA-Fe, $\mathrm{Cu}$, and Mn did not exhibit similar correlations with yield and uptake during the 2011 barley crop season and may indicate factors other than availability during the early stages may influence total plant uptake of minor elements. These factors may include the amounts of total and available minor elements in the studied soil. For example, Obrador et al. (2007) reported that SOM can increase the availability of $\mathrm{Zn}$ and $\mathrm{Mn}$ to barley plants. Soil organic matter chelates these minor elements and increases desorption of metals from soils and keep them in solution for uptake by plant roots (Lindsay and Norvell, 1978). DTPA is known to chelate $\mathrm{Fe}, \mathrm{Zn}, \mathrm{Cu}$, or Mn even in the presence of $\mathrm{Ca}^{2+}$ and $\mathrm{Mg}^{2+}$ in soil solution (Lindsay and Norvell, 1978), a geochemical situation that holds true in our study. Critical levels of DTPA-Fe, $\mathrm{Cu}$, and $\mathrm{Mn}$ for sorghum are 2.4-4.5, 1.2, $0.2 \mathrm{mg}$ DTPA-Fe, $\mathrm{Mn}$, and $\mathrm{Cu}$ $\mathrm{kg}^{-1}$ soil, respectively (Lindsay and Norvell, 1978). The inherent contents of DTPA-Fe, $\mathrm{Mn}$, and $\mathrm{Cu}$ in our study are above these critical levels, and may have explained the inconsistent correlations between minor element contents, biomass yields and plant uptake in the 2011 barley crop season. Other factors that may play roles in the uptake of minor nutrients include CEC where positively-charged minor elements can be adsorbed on the exchange complex in soils. The contents of minor nutrients in barley reported in this study falls within the following contents $\left(\mathrm{mg} \mathrm{kg}^{-1}\right)$ of minor nutrients for five cultivars of barley observed by Dick et al. (1985): Zn (25-30), Fe (73-89), Cu (4.7-5.6), and Mn (28-39).

\section{CONCLUSIONS}

It can be concluded that two-time additions of pig slurry at rate of 170 and $340 \mathrm{~kg} \mathrm{~N}^{-1}$ increased major (N, P, K, $\mathrm{Mg}$ ) and minor ( $\mathrm{Zn}, \mathrm{Fe}, \mathrm{Cu}, \mathrm{Mn})$ nutrients in soils planted to barley. We recommend the application of pig slurry at 1-mo prior to crop cultivation to best assess the amounts of available $\mathrm{NO}_{3}^{-}-\mathrm{N}$, Olsen-P, DTPA-Zn, Fe, $\mathrm{Cu}$, and $\mathrm{Mn}$ that correlate well with biomass yield and nutrient uptake. The agronomic benefits (yield and uptake) to barley justify the utility of pig slurry application to agricultural soils in addition to being an environmentally-friendly way to manage the ever-increasing amounts of the generation of pig slurry. However, it is recommended that monitoring of composition of leachates from agricultural soils treated with pig slurry should be undertaken to safeguard the 
groundwater from nitrate build up and/or eutrophication due to accumulations of phosphate.

\section{ACKNOWLEDGEMENTS}

We thank the financing of this study by the Research Group: Sustainable Use, Management and Reclamation of Soil and Water from de Technical University of Cartagena (Spain). JMA wishes to acknowledge the support of Natural Sciences and Research Council (Canada) and the Ministry of Education (Spain) during his sabbatical leave at the Technical University of Cartagena (Spain).

\section{LITERATURE CITED}

APHA, AWWA, and WPCF. 1992. Standard methods for the examination of water and wastewater. $20^{\text {th }}$ ed. American Public Health Association, American Water Works Association and Water Pollution Control Federation Washington, D.C., USA.

Arvidsson, J. 1999. Nutrient uptake and growth of barley as affected by soil compaction. Plant and Soil 208:9-19.

Ayuso, M., J.A. Pascual, C. García, and T. Hernandez. 1996. Evaluation of urban wastes for agricultural use. Journal Soil Science and Plant Nutrition 42:105-111.

Bernal, M.P., A. Roig, and D. Garcia. 1993. Nutrient balances in calcareous soils after application of different rates of pig slurry. Soil Use and Management 9:9-14.

Biau, A., F. Santiveri, I. Mijangos, and J. Lloveras. 2012. The impact of organic and mineral fertilizers on soil quality parameters and the productivity of irrigated maize crops in semiarid regions. European Journal of Soil Biology 53:56-61.

Burt, R. (ed.) 2004. Soil survey laboratory manual. Soil Survey Investigations Report nr 42 Version 4.0 November 2004. USDANRCS, Lincoln, Nebraska, USA.

Cavanagh, A., M.O. Gasser, and M. Labrecque. 2011. Pig slurry as fertilizer on willow plantation. Biomass and Bioenergy 35:41654173.

Cela, S., F. Santiveri, and J. Lloveras. 2011. Residual effects of pig slurry and mineral nitrogen fertilizer on irrigated wheat. European Journal of Agronomy 34:257-262.

Chantigny, M.H., P. Rochette, and D.A. Angers. 2001. Short-term $\mathrm{C}$ and $\mathrm{N}$ dynamics in a soil amended with pig slurry and barley straw: a field experiment. Canadian Journal of Soil Science 81:131-137.

Daudén, A., and D. Quílez. 2004. Pig slurry versus mineral fertilization on corn yield and nitrate leaching in a Mediterranean irrigated environment. European Journal of Agronomy 21:7-19.

Dick, A.C., S.S. Malhi, P.A. O'Sullivan, and D.R. Walker. 1985. Chemical composition of whole plant and grain and yield of nutrients in grain of five barley cultivars. Plant and Soil 86:257264.

Duchaufour, P.H. 1970. Precis de pedologie. p. 257-280. Masson, Paris, France.

Eurostat. 2010. Censo de ganado porcino en Europa 2010.3tres3. La página del cerdo. Statistical Office of the European Communities, Luxembourg. Available at http://www.3tres3.com/buscando/ censo-de-ganado-porcino-en-europa-2010_3308/ (accessed 20 April 2011).

Gagnon, B., R.R. Simard, R. Robitaille, M. Goulet, and R. Rioux. 1997. Effect of composts and inorganic fertilizers on spring wheat growth and N uptake. Canadian Journal of Soil Science 77:487495.

Gardiner, D.T., and R.W. Miller. 2001. Soils in our environment. $10^{\text {th }}$ ed. Pearson-Prentice Hall, New Jersey, USA
Gilly, J.E., and B. Eghball. 2002. Residual effects of compost and fertilizer applications on nutrients in runoff. Transactions of the American Society of Agricultural Engineers 45:1905-1910.

Jeffery, G.H., J. Basset, J. Mendham, and R.C. Denney. 1989. Vogel's textbook of quantitative chemical analysis. p. 702-703. Longman Scientific \& Technical, Bath, UK.

Kandeler, E., M. Stemmer, and E.M. Klimanek. 1999. Response of soil microbial biomass. Urease and xylanase within particle size fraction to long-term management. Soil Biology and Biochemistry 31:261-273.

Keeney, D.R., and D.W. Nelson. 1982. Nitrogen inorganic forms. In Methods of soil analysis. Part 2. Chemical and microbiological properties. $2^{\text {nd }}$ ed. Agronomy 9:643-698.

Kuligowski, K., T.G. Poulsen, P. Rubæk, and G.H. Sørensen. 2010. Plant-availability to barley of phosphorus in ash from thermally treated animal manure in comparison to other manure based materials and commercial fertilizer. European Journal of Agronomy 33:293-303.

Lindsay, W.L., and W.A. Norvell. 1978. Development of a DTPA soil test for zinc, iron, manganese, and copper. Soil Science Society of America Journal 42:421-428.

Madrid, A., R. Madrid, and J.M. Vicente. 1996. Fertilizantes. 436 p. AMV Ediciones y Mundi-Prensa, Madrid, España.

MARM. 2008. Resultados Encuesta Ganado Porcino Diciembre de 2008. Ministerio de Medio Ambiente y Medio Rural y Marino (MARM), Madrid, España. Available at http://www.magrama. gob.es/es/estadistica/temas/estadisticas-agrarias/2008-12 Porcino_Resultados_tcm7-14366.pdf (accessed 20 April 2011).

MARM. 2010. Ministerio de Medio Ambiente y Medio Rural y Marino (MARM), Madrid, España. Censo de ganado porcino en Europa 2010. 3tres3.com La página del cerdo. Available at http://www.3tres3.com/buscando/censo-de-ganado-porcino-eneuropa-2010_3308/(accessed 20 April 2011).

Martínez, J., and C. Burton. 1998. Processing strategies for farm livestock slurries to minimize pollution and to maximize nutrient utilization: a European perspective. p. 179-189. In T. Matsunaka (ed.) Proceeding of the International Workshop on Environmentally Friendly Management or Farm Animal Waste.

Nielsen, N.E., and E.M. Hansen. 1984. Macronutrient cation uptake by plants. II. Effects of plant species, nitrogen concentration in plant, cation concentration, activity and activity ratio in solution. Plant and Soil 77:347-365.

Nielsen, N.E., and H.E. Jensen. 1986. The course of nitrogen uptake by spring barley from soil and fertilizer nitrogen. Plant and Soil 91:391-395.

Obrador, A., J.M. Alvarez, L.M. Lopez-Valdivia, D. Gonzalez, J. Novillo, and M.I. Rico. 2007. Relationships of soil properties with $\mathrm{Mn}$ and $\mathrm{Zn}$ distribution in acidic soils and their uptake by a barley crop. Geoderma 137:432-443.

Olsen, S., C. Cole, F. Watanabe, and L. Dean. 1954. Estimation of available phosphorus in soils by extraction with sodium bicarbonate. USDA Circular nr 939. 19 p. USDA, Washington, D.C., USA.

Pan, W.L., and A.G. Hopkins. 1991. Plant development, and N and $\mathrm{P}$ use of winter barley II Response to tillage and $\mathrm{N}$ management across eroded toposequence. Plant and Soil 135:21-29.

Real Decreto 324/2000. 2000. Real Decreto 324/2000, de 3 de marzo, por el que se establecen normas básicas de ordenación de las explotaciones porcinas. BOE 58, Agencia Estatal Boletín Oficial del Estado, Madrid, España. Available at http://www.boe. es/diario_boe/txt.php?id=BOE-A-2000-4447 (accessed 20 April 2011).

Richards, L.A. 1954. Diagnosis and improvement of saline and alkaline soils. Agricultural handbook 60. US Department of Agriculture (USDA), Washington, D.C., USA.

Sempere, A., J. Oliver, and C. Ramos. 1993. Simple determination of nitrate in soils by second-derivative spectroscopy. Journal of Soil Science 44:633-639.

Sieling, K., T. Brase, and V. Svib. 2006. Residual effects of different $\mathrm{N}$ fertilizer treatments on growth, $\mathrm{N}$ uptake and yield of oilseed 
rape, wheat and barley. European Journal of Agronomy 25:40-48. Soon, Y. 1988. Nutrient uptake by barley roots under field conditions Plant and Soil 109:171-179.

Soumare, M., F.M.G. Tack, and M.G. Verloo. 2003. Effects of a municipal solid waste compost and mineral fertilization on plant growth in two tropical agricultural soils of Mali. Bioresource Technology 86:15-20.

StatSoft Inc. 2011. STATISTICA (data analysis software system), version 10. Available at www.statsoft.com (accessed 20 April 2011).

Stevenson, F.C., A.M. Johnston, H.J. Beckie, S.A. Brandt, and L. Townley-Smith. 1998. Cattle manure as a nutrient source for barley and oilseed crops in zero and conventional tillage systems. Canadian Journal of Plant Science 78:409-416.

USDA. 2010. Keys to soil taxonomy. $11^{\text {th }}$ ed. US Department of Agriculture (USDA), Natural Resources Conservation Service, Washington, D.C., USA.

Walkley, A., and I.A. Black. 1934. An examination of the Degtjareff method for determining organic carbon in soils: Effect of variations in digestion conditions and of inorganic soil constituents. Soil Science 63:251-263

Whalen, S.C., and J.T. DeBerardinis. 2007. Nitrogen mass balance in fields irrigated with liquid swine waste. Nutrient Cycling in Agroecosystems 78:37-50.

Yagüe, M.R., A.D. Bosch-Serra, M. Antúnez, and J. Boixadera. 2012. Pig slurry and mineral fertilization strategies' effects on soil quality: Macroaggregate stability and organic matter fractions. Science of the Total Environment 438:218-224

Zanuzzi, A., J.M. Arocena, J.M. van Mourik, and A. Faz. 2009 Amendments with organic and industrial wastes stimulate soil formation in mine tailings as revealed by micromorphology. Geoderma 154:69-75.

Zornoza, R., A. Faz, D.M. Carmona, S. Kabas, S. Martinez-Martinez, and J.A. Acosta. 2012. Plant cover and soil biochemical properties in a mine tailing pond five years after application of marble wastes and organic amendments Pedosphere 22:1-11. 